\title{
Commentary
}

\section{Important Differences Between Topoisomerase-I and -II Targeting Agents}

\section{Shantanu Banerii \\ Marek Los*}

Manitoba Institute of Cell Biology; Winnipeg, Manitoba, Ontario Canada

*Correspondence to: Marek Los; Canada Research Chair in New Cancer Therapy Development; Manitoba Institute of Cell Biology; 675 McDermot Avenue; Room ON 6010; Winnipeg, MB R3E OV9 ; Tel.: 204.787.2294; Fax: 204.787.2190; Email: losmi@cc.umanitoba.ca

\section{Received 08/14/06; Accepted 08/14/06}

This manuscript has been published online, prior to printing, for Cancer Biology \& Therapy Volume 5, Issue 8. Definitive page numbers have not been assigned. The current citation for this manuscript is: Cancer Biol Ther 2006; 5(8):

http://www.landesbioscience.com/iournals/cbt/abstract.php?id = 3274

Once the issue is complete and page numbers have been assigned, the citation will change accordingly.

\section{KEY WORDS}

AUTHOR: PLEASE PROVIDE 5-7 KEY WORDS

Commentary to:

Activation of ATM and Histone H2AX Phosphorylation Induced by Mitoxantrone But Not by Topotecan is Prevented by the Antioxidant N-acetyl-L-Cysteine

Xuan Huang, Akira Kurose, Toshiki Tanaka, Frank Traganos, Wei Dai and Zbigniew Darzynkiewicz

Cancer Biology \& Therapy 2006; 8:In press
The twentieth century marked the discovery of numerous drugs with varying degrees of efficacy against rapidly dividing cancer cells. In the 1950's, the first of a new class of drugs, camptothecin was found to be a strong inhibitor of DNA synthesis. ${ }^{1}$ Unfortunately, this early formulation had poor aqueous solubility and severe clinical toxicity. Years later, these solubility issues were corrected and more tolerable formulations such as topotecan were designed. ${ }^{2,3}$ The paper by Huang and colleagues compares the molecular mechanism of action between a topoisomerase-I inhibitor topotecan, and a topoisomerase-II inhibitor mitoxantrone, pointing at important, therapy-relevant differences in their mechanism of action. $^{4}$

Despite demonstrated clinical efficacy, the exact target of DNA-synthesis inhibitors remained elusive until the 1980 's, when reports emerged that camptothecin and its analogues interacted with DNA topoisomerase-I. ${ }^{5,6}$ These topoisomerase enzymes have the ability to relax and untangle large strands of DNA by the process of transesterification. ${ }^{7}$ Generation of this transient 'cleavable complex' relieves the torsional stress that develops in the DNA helix during replication and transcription. ${ }^{7}$ It is proposed that drugs which interact with topoisomerase stabilize this cleavable complex and induce double-stranded DNA breaks upon collision of this complex with the moving replication fork, thereby ultimately leading to cell cycle arrest and apoptosis. ${ }^{2,3,8}$ These topoisomerase-I targeting drugs appear more specific to the $S$ or DNA synthesis specific phase of the cell cycle. ${ }^{2,3}$

While topoisomerase-I causes single-strand DNA breaks, topoisomerase-II itself induces transient double-strand DNA breaks. Topoisomerase-II is crucial for chromosome condensation and segregation, and cells that lack this enzyme are rendered unviable., 9 Drugs such as doxorubicin and its analogue mitoxantrone partially exert their effect by targeting topoisomerase-II. ${ }^{9,10}$ Unlike topoisomerase-I targeting drugs, topoisomerase-II inhibitors exert their effect throughout the cell cycle likely by interfering with both DNA and RNA polymerases. ${ }^{10}$ Despite similar structures, mitoxantrone and doxorubicin have quite different levels of efficacy against common tumors. Mitoxantrone has a very narrow spectrum of activity restricted to breast, prostate, acute leukemia, and lymphoma, whereas doxorubicin has been proven to be active against a broad range of cancers ranging from numerous leukemic cell types to practically all solid organ tumors. ${ }^{3,10}$ The toxicity profile, although similar, is far more dramatic for doxorubicin, particularly with respect to irreversible cardiomyopathy. These clinical toxicities are partly explained by doxorubicin's known effects such as DNA intercalation, inhibition of helicases, generation of reactive oxygen species (ROS), release of cellular iron, and stabilization of topoisomerase II. ${ }^{9,10}$ As revealed by its clinical efficacy, mitoxantrone's mechanism of action, although likely similar to that of doxorubicin, has so far been less clear. It is unclear, for example, whether mitoxantrone exerts its effects in a similar manner, particularly with respect to ROS formation.

Virtually all cells in various stages of growth develop potentially lethal double-strand DNA breaks. Preventing the demise of the cell is a network of proteins, which rapidly detect this damage and activate a series of steps, which stall cell-cycle progression and initiate repair of the DNA. A central protein to the detection of these DNA breaks and initiation of repair is a nuclear serine-threonine kinase called ataxia-telangiectasia mutated (ATM). ${ }^{11,12}$ Upon formation of a double strand break the Mre11-Rad50-Nbs1 (MRN) complex is activated which in turn recruits ATM dimers. ${ }^{12}$ These dimers are phosphorylated at the Serine-1981 position forming active ATM monomers, which in turn activate the checkpoint kinases Chk1 and Chk2, which cause cell-cycle arrest, partly through their effects on p53 and p21. ${ }^{11}$ Also observed early during the formation of double-strand breaks is the phosphorylation of histone H2AX and the detection of $\gamma-\mathrm{H} 2 \mathrm{AX}$ at the site of DNA breaks. ${ }^{13}$ Recently a model utilizing ATM $^{-/}$cell lines has shown that ATM plays a key role in the $\gamma$-H2AX generation. ${ }^{14}$ 
In their recent study, Huang and colleagues have examined the role of ROS in the induction of DNA damage by both the topoisomerase-I interacting drug topotecan and the topoisomerase-II interacting drug mitoxantrone. ${ }^{4}$ As a more sensitive measure of doublestranded DNA breaks, they have utilized concurrent immunocytochemical detection of both activated ATM and $\gamma-\mathrm{H} 2 \mathrm{AX}$ in treated human promyelocytic leukemic (HL-60) and pulmonary carcinoma (A549) cells. ${ }^{15,16}$ As in previous studies, the authors were able to demonstrate increased detection of $\gamma$-H2AX in HL-60 cells within one hour of exposure to either topotecan or mitoxantrone. Again, topotecan induced $\gamma$-H2AX was most evident in S-phase cells whereas mitoxantrone induced $\gamma-\mathrm{H} 2 \mathrm{AX}$ in all phases of the cell cycle. Similar observations were made with the detection of activated ATM. In both cases, only S-phase cells underwent apoptosis upon exposure to either drug. In the presence of $\mathrm{N}$-acetyl-L-cysteine (NAC), a potent ROS scavenger, both HL-60 and A549 had attenuated $\gamma$-H2AX induction and ATM activation in response to mitoxantrone. By contrast, topotecan produced slightly increased rates of $\gamma-\mathrm{H} 2 \mathrm{AX}$ and ATM activation in the presence of NAC.

This study broadens our understanding of the mechanism of action of topotecan and mitoxantrone. The elegantly designed and well controlled experiments show that topotecan effectively targets cells actively replicating DNA, likely through its stabilization of the cleavable complex of topoisomerase-I, and that there does not appear to be any role for the activation of ROS in the anti-tumor activity of this drug. Thus, the limiting factor of topotecan's clinical efficacy remains the fact that cells not actively replicating their DNA are not as sensitive to topotecan and this likely leads to disease progression or relapse. Potentially driving cells into $S$-phase may resolve this barrier. In contrast, mitoxantrone, which targets topoisomeraseII, appears also to act in a ROS-dependent manner. Thus, it causes the double-strand DNA break formation by two different mechanisms; as a result of topoisomerase-II-blockage and by ROS-dependent manner. ROS probably accounts for about $1 / 3$ of mitoxantrone's DNA-damaging capacity, since NAC is able to attenuate less than one third of the total observed double-strand DNA breaks upon mitoxantrone treatment. Similar attenuation of double-strand breaks upon pretreatment with NAC and exposure to other established topoisomerase-II targeting drugs, like doxorubicin and newer agents like salvacine have been reported previously, but the mechanism of ROS generation in this metabolic context is not fully understood. ${ }^{17,18}$ Mitoxantrone is not unique in its capacity as a ROS-generation facilitator, and this quality is shared with other groups of drugs, for example farnesyl-transferase inhibitors. ${ }^{19}$ A limiting factor in the use of topoisomerase-II targeting drugs is the significant toxicity that develops with the cumulative use of these drugs. Thus, there is an urgent need for the development of future treatment regimens that may reduce the total dose of these drugs by combining them with inhibitors of DNA repair, currently under investigation. ${ }^{18-20}$ The study by Huang and colleagues provides important new insight into the molecular mechanism of action of these drugs, thus facilitating the development of new therapy protocols.

\section{References}

1. Wall MEW, M.C.; Cook C.E.; et al. Plant antitumor agents, I: the isolation and structure of camptothecin, a novel alkaloidal leukemia and tumor inhibitor from Camptotheca acumunita. J Am Chem Soc 1966; 88:3888-90.

2. Sparreboom AZ, W.C. Topoisomerase I-Targeting Drugs. In: Chabner BAL, D.L., editor. Cancer Chemotherapy \& Biotherapy: Principles and Practice. 4th ed. Philadelphia: Lippincot Williams \& Wilkins; 2006
3. Takimoto CH. Topoisomerase Interactive Agents. In: Devita VTH, S.; Rosenberg, S.A., editor. CANCER: Principles \& Practice of Oncology. 7th ed. Philadelphia: Lippincott Williams \& Wilkins; 2005.

4. Huang X, Kurose A, Tanaka T, Traganos F, Dai W, Darzynkiewicz Z. Activation of ATM and Histone H2AX Phosphorylation Induced by Mitoxantrone But Not by Topotecan is Prevented by the Antioxidant N-acetyl-L-Cysteine. Cancer Biol Ther 2006; 5:In press.

5. Hsiang YH, Hertzberg R, Hecht S, Liu LF. Camptothecin induces protein-linked DNA breaks via mammalian DNA topoisomerase I. J Biol Chem 1985; 260:14873-8.

6. Hsiang YH, Liu LF. Identification of mammalian DNA topoisomerase I as an intracellular target of the anticancer drug camptothecin. Cancer Res 1988; 48:1722-6.

7. Wang JC. Cellular roles of DNA topoisomerases: a molecular perspective. Nat Rev Mol Cell Biol 2002; 3:430-40.

8. Hsiang YH, Lihou MG, Liu LF. Arrest of replication forks by drug-stabilized topoisomerase I-DNA cleavable complexes as a mechanism of cell killing by camptothecin. Cancer Res 1989; 49:5077-82.

9. Pommier YG, F. Topoisomerase II Inhibitors: The Epipodophyllotoxins, Acridines, and Ellipticines. In: Chabner BAL, D.L., editor. Cancer Chemotherapy \& Biotherapy: Principles and Practice. 4th ed. Philadelphia: Lippincot Williams \& Wilkins; 2006.

10. Doroshow JH. Anthracyclines and Anthracenediones. In: Chabner BAL, D.L., editor Cancer Chemotherapy \& Biotherapy: Principles and Practice. 4th ed. Philadelphia: Lippincot Williams \& Wilkins; 2006.

11. Abraham RT, Tibbetts RS. Cell biology. Guiding ATM to broken DNA. Science 2005; 308:510-1.

12. Zhou BB, Elledge SJ. The DNA damage response: putting checkpoints in perspective. Nature 2000; 408:433-9.

13. Modesti M, Kanaar R. DNA repair: spot(light)s on chromatin. Curr Biol 2001; 11:R22932 .

14. Burma S, Chen BP, Murphy M, Kurimasa A, Chen DJ. ATM phosphorylates histone H2AX in response to DNA double-strand breaks. J Biol Chem 2001; 276:42462-7.

15. Banath JP, Olive PL. Expression of phosphorylated histone H2AX as a surrogate of cell killing by drugs that create DNA double-strand breaks. Cancer Res 2003; 63:4347-50.

16. Tanaka T, Kurose A, Huang X, Dai W, Darzynkiewicz Z. ATM activation and histone $\mathrm{H} 2 \mathrm{AX}$ phosphorylation as indicators of DNA damage by DNA topoisomerase I inhibitor topotecan and during apoptosis. Cell Prolif 2006; 39:49-60.

17. Kurz EU, Douglas P, Lees-Miller SP. Doxorubicin activates ATM-dependent phosphorylation of multiple downstream targets in part through the generation of reactive oxygen species. J Biol Chem 2004; 279:53272-81.

18. Lu HR, Zhu H, Huang M, Chen Y, Cai YJ, Miao ZH, Zhang JS, Ding J. Reactive oxygen species elicit apoptosis by concurrently disrupting topoisomerase II and DNA-dependent protein kinase. Mol Pharmacol 2005; 68:983-94.

19. Pan J, She M, Xu ZX, Sun L, Yeung SC. Farnesyltransferase inhibitors induce DNA damage via reactive oxygen species in human cancer cells. Cancer Res 2005; 65:3671-81.

20. Willmore E, de Caux S, Sunter NJ, Tilby MJ, Jackson GH, Austin CA, Durkacz BW. A novel DNA-dependent protein kinase inhibitor, NU7026, potentiates the cytotoxicity of topoisomerase II poisons used in the treatment of leukemia. Blood 2004; 103:4659-65. 